\title{
Evolution of Symbolisation: Signposts to a Bridge between Connectionist and Symbolic Systems
}

\author{
Ronan G. Reilly \\ Department of Computer Science \\ National University of Ireland, Dublin \\ Ronan.Reilly@ucd.ie
}

\begin{abstract}
This paper describes recent attempts to understand the evolution of language in humans and argues that useful lessons can be learned from this analysis by designers of hybrid symbolic/connectionist systems.
\end{abstract}

\section{Introduction}

If one doesn't subscribe to the theory of the serendipitous emergence of language in our ancestors several hundred thousand years ago, one is faced with a conundrum. If language has been the product of evolution, what were its intermediate stages, what foundation was it constructed upon? This problem has recently been thoroughly analysed by Terrence Deacon (1997) in his book "The Symbolic Species". Deacon argues for the existence of an evolutionary path in the development of the relationship between sign and signified that proceeds from an iconic relationship, through a process of temporal and spatial indexicalisation (i.e., association), to an arbitrary and culturally licensed symbolic one.

The implications that this perspective has for hybrid symbolic/connectionist system will be explored here.

\section{An Evolutionary Perspective}

Similarly to C.S. Pierce, Deacon distinguishes between three categories of sign: iconic, indexical, and symbolic. Iconic signs have some physical similarity with what they signify, indexical signs are related to their referent either spatially or temporally, and symbolic signs have an arbitrary relationship with their referent. In keeping with an evolutionary perspective, Deacon makes the case that symbol systems emerged in human species through a process that moved from iconic through indexical to symbolic sign usage. Each succeeding level of sign usage subsumed the preceding one. Thus indexical signs are built upon spatio-temporal relationships between icons, and symbols are constructed upon relationship between indices and most importantly on relationship between other symbols. So for example, a child learning to read, first encounters printed words as iconic of print in general, much in the same way as someone who doesn't read Chinese might look upon a book of Chinese characters. Each character is equally iconic (trivially) of 
written language. As the child learns the writing system she proceeds to a stage where the written signs index the spoken language and her perceptual environment in a systematic way. Finally, the relationships among these indices allow her to access the symbolic aspects of the words. However, the written words acquire their symbolic status not simply by standing for something, but from the mesh of intersymbolic relationships in which they are embedded.

One of Deacon's central points is that there is an isomorphism between the stages leading to symbol development and the transition from perception (of icons), through associationistic learning (of indices), through cognising (of symbols). Only humans have reached the final stage, though Deacon argues that the bonobo chimpanzee, Kanzi (Savage-Rumbaugh \& Lewin, 1994), has attained a level of sophistication in the symbolic domain that poses a major challenge to language nativists. Overall, Deacon's thesis is an alternative to the nativists' arguments (Chomsky, 1986; Pinker, 1994) for the origins of language. According to Deacon, symbolic cognition, of which language is just one manifestation, emerged as a function of an evolutionary dynamic between the selectional advantages provided by simple symbol usage and pre-frontal cortical changes that favoured and supported symbol usage.

Deacon supports his case by looking at the comparative neural anatomy of apes and humans. He demonstrates that, contrary to conventional wisdom, brain size is not the key inter-species difference. Rather it is the disproportional growth of the pre-frontal cortex relative to other cortical areas. The influence that this area has over the rest of the cortex, as indicated by the cortico-cortical projections emanating from, and projecting to, the region suggests that it plays a significant role in the acquisition of symbolic behaviour. An example of the role of the pre-frontal cortex in a natural primate environment would be its use in foraging behaviour. In foraging, an effective strategy is not to return to the locations that one has most recently visited, since they are least likely to provide a food reward. Thus, one needs to be able to suppress the more basic drive to return to where one has previously had one's behaviour reinforced.

In humans the pre-frontal enlargement dominates cortical function, and biases us to a specific style of learning. This involves, among other things, the ability to detach from the immediate perceptual demands of a task, to switch between alternative courses of action, to delay an immediate response to a stimulus, to tune into higher-order features of the stimulus environment, and so on. All of these capabilities are essential pre-requisites for a facility with symbols.

\section{A Maturational Wave}

One of the paradoxes of artificial neural network research is that the capabilities of artificial neural networks fall far short of those of the real thing, yet the learning algorithm(s) employed by real neural networks may well be considerably simpler and less powerful than, say, error backpropagation (Rumelhart, Hinton, \& Williams, 1986). The evidence to date suggests that some variant of Hebb's rule, possibly mediated by NMDA-based long term potentiation, may be the dominant learning rule in natural nervous systems (Cruikshank \& Weinberger, 1996). The problem is that Hebb's rule cannot be used to learn even a trivial higher order function such as 
XOR, at least not directly. So this presents the evolutionary account of the emergence of symbolisation with a problem. If the symbolic system is indeed built upon layers of indexical and iconic relationships, we don't have, prima facie, a biological learning algorithm that is up to the job of acquiring the type of secondorder features of the environment necessary for symbol use. Not only do we have an impoverishment of stimulus if the nativist position is to be believed, we also appear to have an impoverishment of learning mechanism.

However, Shrager and Johnson (1996) in an elegant computational study demonstrated that the inclusion of a wave of learning plasticity passing through a model cortex permitted the acquisition of higher-order functions using simple Hebbian learning. There is good evidence that just such a modulation of plasticity occurs during cortical development (Thatcher, 1992). An important feature is that it affects the sensory-motor regions of the cortex initially and then moves through to regions more distal from sensory-motor areas. Elman and Rebotier (1997) argued that this may have implications for language learning, in that it demonstrated an interaction between developing cognitive capacity and the acquisition of rule-like behaviour of increasing complexity. In an earlier study, Elman (1993) had shown that while a simple recurrent network (SRN) could not learn a complex context-free grammar without an initial limitation in its memory capacity, which was gradually enhanced. This gradual increase in memory capacity was analogous to that found in developing children.

There seems to me to be a useful connection to be made between Deacon's argument for the evolution and emergence of symbolic behaviour and Shrager et al.'s discovery of relationship between developmental plasticity and the acquisition of higher-order functions. On the one hand Deacon proposes that the emergence of language coincides with the ability to abstract higher-order features of the environment mediated by an expansion of the pre-frontal cortex, while on the other hand Shrager et al. demonstrate with their computational model that a wave of learning plasticity travelling from primary sensory-motor areas to more distal regions can permit the development of higher-order functions using just Hebbian learning. The connection between these two observations is that the pre-frontal cortex is one of the later regions to experience the wave of plasticity, and is therefore implicated in the development of higher-order functions. It is quite likely, therefore, that these higher-order functions are the very ones that are significant in the development of linguistic and general symbolic capabilities of humans.

\section{Symbol Emergence}

Deacon identifies the case of Kanzi (Savage-Rumbaugh \& Lewin 1994) as suggestive of how symbol use might have got started in an early hominid species such as Australopithecus. Kanzi acquired the ability to use an artificial language involving a board of lexigrams while being cared for by his mother. One of the more interesting features of this case is that Kanzi was not explicitly taught how to use the lexigrams himself, but acquired his ability incidentally while his mother was going through a training regime that ultimately proved ineffective for her. This appears to be analogous to the phenomenon described by Elman (1993), where a complex grammar could only be acquired by a connectionist network by "filtering" 
it through an initially limited memory capacity, akin to the capacity limitations we find with children. What is important from an evolutionary point of view, however, is not the means by which Kanzi acquired a significant facility with symbols, but that he was able to do so at all. This suggests that somewhere back in hominid evolution conditions prevailed that facilitated and favoured the expression of an incipient symbol ability. This then set in train a process of dynamical co-evolution between brain structure and cognitive abilities that led to the emergence of the complex cognitive and linguistic skills that we manifest as a species.

The evolutionary jump to symbolisation from an association-based indexical system, argued for by Deacon, requires an ability to discern certain key relationships between symbols. Although not referred to by Deacon (1997) explicitly as such, these relationships are primarily those of compositionality and systematicity. These are the very features identified by Fodor and Pylyshyn (1988) as noticeably absent from connectionist approaches to language and cognition. While the compostionality criticism has been positively addressed to most people's satisfaction by Van Gelder (1990), systematicity still remains a keenly debated issue (Hadley, 1994). In the case of connectionist NLP models, it boils down to their ability (or lack of it) to generalise their behaviour to new words in new positions in test sentences. Notwithstanding some positive indications (Christiansen \& Chater, 1994), current connectionist models do not as yet demonstrate the strong systematicity characteristic of a human language user. Something is missing from these connectionist accounts, and a clue to what this might be can be had by exploring the role of the prefrontal cortex in human language use.

\section{Signposts to a Connectionist/Symbolic Bridge}

So what are the signposts referred to in the title of this paper? While I cannot suggest an immediate solution to the problem of figuring out how best to integrate symbolic and connectionist modes of computation, I do believe that an analysis of developments in language evolution provides useful indicators of where we should look for promising approaches. Some of the indications are that:

(1) we should build complex hybrid systems from simpler connectionist ones using a maturational dynamic;

(2) we should explore the use of simple learning rules, such as Hebbian learning, in conjunction with maturation;

(3) we should focus on finding ways to resolve the limitations in systematicity of connectionist models;

I believe that the evidence adduced by Deacon indicates that while symbolisation is constructed upon an iconic and associationistic foundation, its emergence has required the addition of a qualitatively different cognitive mechanism. In light of this, the view that symbolisation is an emergent (or even epi-) phenomenon from a non-symbolic substrate is untenable. On the other hand, the additional mechanism is not a language-specific alteration to the cognitive architecture, but a generic modification that cuts across all aspects of cognitive function. Explorations in the space of hybrid symbolic/connectionist models that are constrained by these assumptions will, I believe, yield fruitful results. 


\section{References}

Chomsky, N. (1986). Knowledge of language. New York: Praeger.

Christiansen, M.H., \& Chater, N. (1994). Generalisation and connectionist language learning. Mind and Language, 9, 273-287.

Cruikshank, S.J., \& Weinberger, N.M. (1996). Evidence for the Hebbian hypothesis in experience-dependent physiological plasticity of neocortex: A critical review. Brain Research Reviews, 22, 191-228.

Deacon, T. (1997). The symbolic species: The co-evolution of language and the human brain. London, UK: The Penguin Group.

Elman, J.L. (1993). Learning and development in neural networks: The importance of starting small. Cognition, 48, 71-99.

Fodor J. A., \& Pylyshyn, Z.W. (1988). Connectionism and cognitive architecture: A critical analysis. Cognition, 28, 3-71.

Hadley, R.F. (1994). Systematicty in connectionist language learning. Mind and Language, 9, 247-271.

Pinker, S. (1994). The language instinct: how the mind creates language. New York: William Morrow.

Rebotier, T.P., \& Elman, J.L., (1996). Explorations with the dynamic wave model. In D. Touretsky, M. Mozer, \& M. Haselmo (Eds.), Advances in Neural Information Processing Systems 8. Cambridge, MA: MIT Press.

Rumelhart, D. E., Hinton, G. E., \& Williams, R. J. (1986). Learning internal representations by error propagation. In D. E. Rumelhart, J. L. McClelland, \& The PDP Research Group (Eds.), Parallel distributed processing. Explorations in the microstructure of cognition. Volume 1: Foundations. Cambridge, MA: MIT Press, pp. 318-362.

Savage-Rumbaugh, E.S., \& Lewin, R. (1994). Kanzi: The ape at the brink of the human mind. New York: John Wiley.

Shrager, J. and M.H. Johnson (1996). Dynamic plasticity influences the emergence of function in a simple cortical array. Neural Networks, 9, 1119-1129.

Thatcher, R.W. (1992). Cyclic cortical reorganization during early childhood. Brain and Cognition, 20, 24-50.

Van Gelder, T. (1990). Compositionality: A connectionist variation on a classical theme. Cognitive Science, 14, 355-384. 